\title{
A Neural Network Based Motor Bearing Fault Diagnosis Algorithm and its Implementation on Programmable Logic Controller
}

\author{
Wedajo T. Abdisa \\ Engineering Institute of Technology, Perth, Australia \\ E-mail: wedajotariku@gmail.com \\ Hadi Harb \\ Engineering Institute of Technology, Perth, Australia \\ E-mail: hadi.harb@eit.edu.au
}

Received: 20 February 2019; Accepted: 20 March 2019; Published: 08 October 2019

\begin{abstract}
This research aims to test the feasibility of Programmable Logic Controller implementation of an Artificial Neural Network based bearing fault diagnosis using vibration datasets. The main drawback of using a Programmable Logic Controller along with an Artificial Neural Network is that it does not support the parallel nature of neural networks. This drawback is not significant for relatively small applications like bearing diagnosis that involve very short execution time. In this paper, a three layer multilayer perceptron backpropagation neural network is trained using Levenberg-Marquardt training algorithm with vibration dataset consisting of four bearing status classes: normal, outer race way fault, inner race way fault and rolling element (ball) fault. Time-frequency domain and time domain input features were considered in this research. Both approaches have performed well during simulation phase. But the time-frequency feature extraction approach was observed to take too long scan cycle time to be implemented in real-time. This is due to the computationally intensive nature of Fast Fourier Transform algorithm involved during feature extraction. The time domain approach is proved to be feasible for Programmable Logic Controller implementation. The time domain input features used for neural network training were root mean square, variance, kurtosis and negative log likelihood values. The average performance obtained during simulation with 10 -fold cross validation performance estimator was an error of $7.9 \times 10^{-4}$. The performance tests of Programmable Logic Controller implementation resulted in $100 \%$ bearing fault detection rate.
\end{abstract}

Index Terms-Artificial neural networks, programmable logic controller, motor bearing, diagnosis, fault detection, vibration data.

\section{INTRODUCTION}

Bearings are essential components of any rotating machines in industrial applications. Electrical motors such as induction motors are one of the most widely used rotating industrial machines that uses roller bearings. The bearings operate under mechanical stress and they are vulnerable to frequent physical faults. These faults cause motor breakdowns that ultimately affect asset performance of a production facility and may also result in personnel injury. The best solution to keep the machine performance high and also avoid personnel casualties and economical loss due to bearing faults is to perform real time continuous fault diagnosis of bearings so that the damage can be detected at an early stage. One of the most effective tools for bearing diagnosis is vibration analysis [4]. Fault features are extracted by signal processing techniques such as Fourier transforms and time domain analysis. The fault features provide information regarding the fault pattern and thus give indication of the fault area. However, most of the currently available techniques require expert knowledge to properly utilize them. On the contrary, most industries need a system that can be understood by operators with relatively low knowledge of such systems $[8,15]$.

Artificial neural networks (ANN) based bearing fault diagnosis technique is an adequate choice in order to automatically and reliably perform the fault diagnosis without the need for a specialist data examination $[8,15]$.

In recent years, the application of neural networks for bearing fault diagnosis is among the research topics of interest by many researchers. A number of papers have been published on the topic with most of them focusing on approach of feature extraction, training algorithm selection and general feasibility of neural network 
application to bearing fault diagnosis [17]. The implementation stage is not widely dealt with at the moment. There are only few research literatures which considered implementation of neural networks for bearing diagnosis. These implementations have considered the use of VLSI (Very Large Scale Integration) devices such as microcontrollers [43, 44] and FPGA (Field Programmable Logic Arrays) [1, 41] to test performance of neural networks at hardware level.

The purpose of this work is to use Programmable Logic Controllers (PLCs), which is the industry standard for control systems, as an implementation hardware and thus introduce neural network-based bearing fault diagnosis application into the industry. The main contributions are summarized as follows:

- Comparing time domain and time-frequency domain features for bearing faults detection in terms of classification accuracy and PLC computing needs.

- Implementing a solution that provides low cost bearing condition monitoring where incipient bearing faults are detected at an early stage before causing machine downtime and also preventing possible personnel causalities.

- Providing a reference for PLC implementation of artificial neural networks that can serve other similar industrial fault diagnosis or control applications.

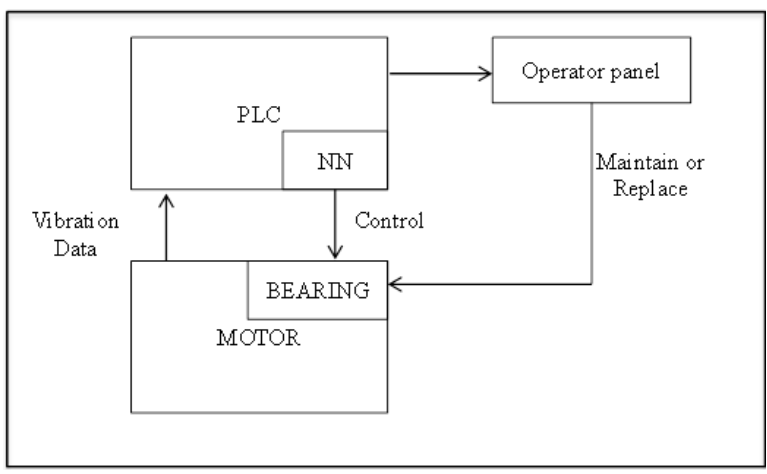

Fig.1. Block diagram showing interconnection of PLC, motor and operator panel

\section{A. Research Method}

The research work deals with both simulation and implementation phases. The main points to be considered as success factors of this work are the performance of the diagnosis system in fault detection and its suitability for PLC implementation.

\section{Data Acquisition Method}

Vibration analysis is the most preferred technique for bearing fault diagnosis as compared to other techniques. It is reliable; it gives clear indication of defect locations; and it is highly sensitive to fault severity levels [17]. The vibration dataset to be used for training neural networks can be obtained from software simulation, laboratory test rigs or from real-time industrial set ups. Most researches are conducted on dataset obtained from computer simulations and laboratory test rigs since real-time industrial vibration data is rarely available $[2,8,15,36$, 37]. In this research, a bearing dataset from bearing data repository of [36] was used. The data was recorded from a test rig consisting of a $2 \mathrm{HP}$ motor, a torque transducer, a dynamometer and control components. Faults of different sizes were seeded into bearings using electrodischarge machining (EDM) at outer race, inner race and rolling element. Data from normal bearing was also recorded for use as baseline data [36]. Selected dataset files are downloaded from the official website of [36]. During real-time applications, accelerometer measurement data is directly stored in PLC data block memory from where the data is retrieved by neural network function block for analysis.

\section{Feature Extraction Method}

The success of a fault diagnosis system highly depends on the amount of relevant information obtained from the selected fault features. Therefore, the choice of feature extraction method is a critical input for neural network training algorithm. The selection of feature extraction method should also consider its feasibility of implementation on PLCs. The performance of timefrequency domain features and time domain features were compared during simulation and implementation phases. Both approaches performed well during simulation phase but time domain approach is the only method where acceptable result was obtained during PLC implementation phase.

\section{Neural Network Archtecture and Training Algorithm}

Multilayer Feedforward Neural Network is the most popular architecture for pattern recognition or classification applications [30, 31, 33]. A three layer feedforward neural network with Levenberg-Marquardt (LM) backpropagation training algorithm on Matlab environment is employed in this study.

\section{PLC Impelementation}

The main purpose of this research work is to experiment and draw conclusion whether PLC implementation of neural networks based bearing fault diagnosis is feasible or not. The key limiting factors in PLCs are CPU scan rate and available memory size. The bearing diagnosing neural network program blocks are intended to be an integral part of the general control blocks sharing the same CPU and memory areas. A virtual PLC called S7-PLCSIM of the Siemens S7-300 family was used for experiments. PLC program for data manipulation, feature extraction and neural networks was developed with SIMATIC Manager software of Siemens S7-300. The raw vibration data representing each category of bearing condition was imported into a PLC data block and analyzed by the programs.

The rest of this paper is organized as follows. A brief overview of related work is presented in Section 2. Section 3 describes the methodology and experimental design. The results are reported in Section 4 and we 
conclude in Section 5.

\section{RELATED WORKS}

Bearing fault detection and diagnosis is one of the pattern recognition and classification problems where artificial neural network finds important industrial applications [8]. Vibration data is the most common and reliable measurement data used for bearing diagnosis in both conventional condition monitoring methods and in most recent approaches $[4,8,10,12,15,16,37]$. The use of motor stator current data is also widely used in recent literatures [2, 7, 20, 21, 22]. According to [17], vibration analysis is the most preferred method as compared to other condition monitoring techniques such as temperature monitoring, acoustic monitoring and wear debris analysis.

The raw vibration data is typically processed to prepare input features using one of the feature extraction techniques. The techniques usually employed are frequency domain analysis [10, 20, 39], time domain analysis [15, 16, 37] or time-frequency analysis [8, 13, 14].

Time domain analysis is the easiest method to analyse vibration signals [40]. It is a method by which statistical features are computed and compared in order to identify a particular fault [37]. These statistical features include mean, standard deviation, variance, root mean square, kurtosis, skewness, crest factor, clearance factor, impulse factor, shape factor, negative log-likelihood and normalized central moments [15, 37]. According to [15], kurtosis and normal negative log-likelihood features performed best on neural network based bearing fault diagnosis. Another similar time domain analysis conducted by [37] using the same source of vibration dataset shows variance and root mean square are the best performing input features.

Time- frequency analysis technique is the use of both time-domain and frequency domain features for bearing diagnosis. It is capable of revealing aspects of data that other signal analysis techniques could not provide by using relevant time domain and frequency domain features together [13]. In [8], peak value, mean value and kurtosis are used along with the characteristic fault frequencies to detect bearing fault based on neural networks.

In this paper we compare time domain and timefrequency domain features with an emphasis on their ease of implementation on a PLC.

\section{METHODOLOGY}

In this section the methods and steps followed in the research work will be discussed. The experimental activities can be categorized into two phases: Matlab based software simulation and PLC implementation phases. Major activities involved from start to finish of the experimental work are as follows:
- searching reliable bearing dataset source and collection of the data

- dataset manipulation and feature extraction

- $\quad$ selection of ANN model and training algorithm

- conducting ANN training and validation experiments using different scenarios

- conversion of trained ANN into the PLC language

- importing bearing dataset into PLC for tests

- comparing PLC implementation results with two different feature extraction approaches

- developing and testing of Graphical User Interface (GUI)

The simulation phase involves training, validation and testing of the neural network. Validation is performed within the training phase using training dataset while testing is conducted with separate testing dataset prepared for this purpose.

\section{A. Selection of data source and collection of dataset}

The dataset used for this experiment was obtained from Case Western Reserve University Bearing Data Center [36]. The dataset consists of normal and defective bearing data. Data for all types of faults with different severity levels at different motor speed was recorded by the bearing data center. This bearing dataset has been validated in many researches and it has become a standard dataset for the study of rolling bearings $[15,35$, 37]. The data was acquired by bearing accelerometers under different motor speed, different operating loads and different bearing conditions [36].

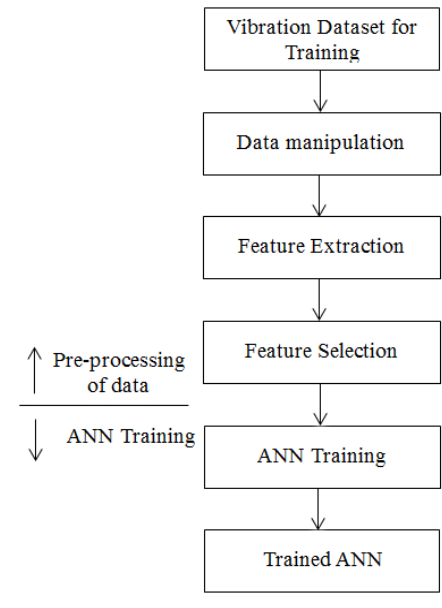

(a) Training phase

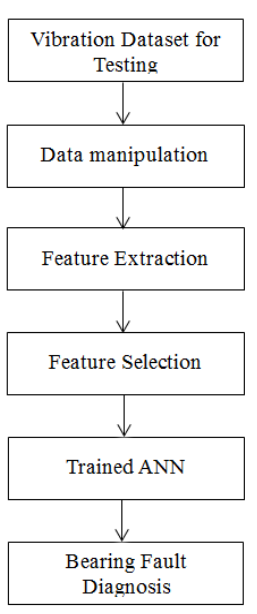

(b) Testing phase
Fig.2. ANN training and testing steps

\section{B. Dataset manipulation and feature extraction}

The dataset collected for each bearing condition was manipulated by Matlab Software in a way suitable for specific feature extraction technique. Thus, training and validation dataset for time-frequency domain feature extraction and time domain feature extraction was prepared separately. 


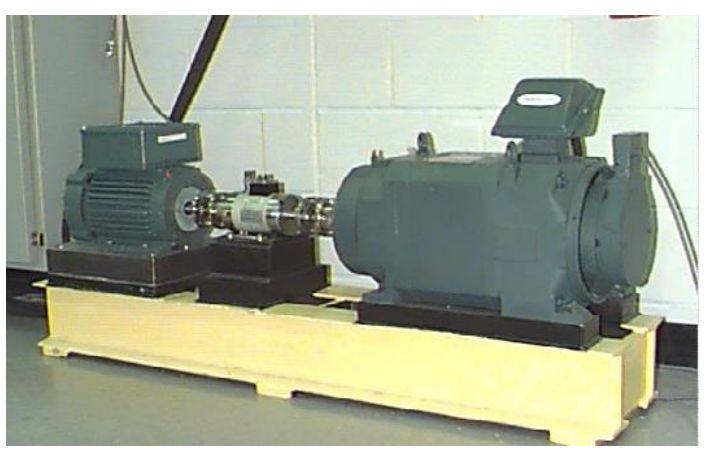

Fig.3. Bearing test rig [36]

\section{Dataset manipulation for time-frequency domain feature extraction approach}

In time-frequency domain feature extraction approach, both time domain features and frequency domain features are used as an input to ANN during training and validation. The features selected are expected to reveal possible faults in roller bearings. In frequency domain, the presence of significant amplitudes of characteristics frequencies within the frequency spectrum of bearing vibration is directly linked to a specific localized bearing fault. The characteristic frequencies are multiples of the shaft frequency as can be seen from below calculations. Thus, due to this link, the use of shaft frequency and its amplitude as input feature can improve training results [8].

Time domain features are statistical values extracted from the datasets. The use of time and frequency domains together is aimed to improve detection and diagnosis accuracy. Time domain values considered in this study for use in combination with frequency domain features were peak amplitude value, mean amplitude value and kurtosis. These time-frequency domain features are proved to give best performance in [8]. The input vector consisting of the selected features is given as:

$$
\mathrm{X}=\left[\begin{array}{llllll}
\text { Amax } & \text { Amean } \mathrm{K} & \mathrm{F}_{\mathrm{S}} & \mathrm{A}_{\mathrm{S}} & \mathrm{A}_{\mathrm{O}} & \mathrm{A}_{\mathrm{I}}
\end{array}\right]
$$

Where Amax represents time domain peak amplitude, Amean represents time domain mean amplitude,

$K$ is the time domain kurtosis,

$F_{S}$ is the shaft frequency,

$A_{S}$ is the amplitude of shaft frequency,

$A_{O}$ is the amplitude of ball pass outer race frequency,

$A_{I}$ is the amplitude of ball pass inner race frequency,

In order to avoid under training of the ANN, a good number of training input vectors are required [7]. Since there are only limited number of datasets available that represents each bearing class, the datasets should be divided into smaller data frames that will be used to prepare a single input vector.

The dataset files of faulty bearings used in this study contained about 120,000 data point recordings at sampling frequency of 12,000 samples per second for 10 seconds. The available dataset for normal bearing was with a file of 480,000 data points recoded over 10 seconds at a sampling frequency of 48,000 samples per second. This normal bearing dataset was first down sampled by 4 to produce a file with 120,000 data points at 12,000 samples per second similar to the faulty bearing datasets for uniformity.

The main point to be considered while determining the minimum number of data points of the data segment for input vector calculation is the frequency resolution [8].

$$
\Delta f=\frac{F_{s a}}{N}
$$

where $F s a$ is the sampling frequency and $N$ is the number of data points.

From equation (1) above, we can observe that the higher the data point results in higher frequency resolution for a constant sampling frequency. Higher frequency resolution implies higher accuracy of characteristic frequency evaluation or higher accuracy of detecting bearing faults.

While working with a limited number of bearing datasets, there should be a balance between number of training input vectors and the number of data points used to compute each input vector. This balance in turn helps to create optimum number of training input data at acceptable level of frequency resolution. To attain this, each dataset file of 120,000 data points was first divided into 3 data segments each with 40,000 data points without changing the sampling frequency. Each of the 3 data segments were then down sampled by 4 where each of these data frames contain 10,000 data points at sampling rate of 3,000 samples per second. Here, using equation (1), the frequency resolution is $0.3 \mathrm{~Hz}$.

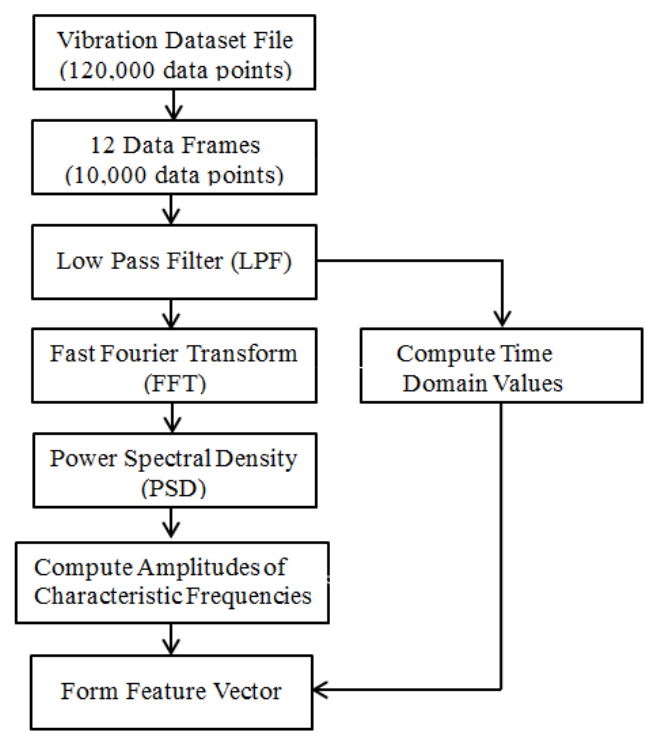

Fig.4. Steps taken to compute time-frequency domain feature vectors

Therefore, we get 12 data frames from each of the original dataset files. These 12 data frames were used to compute 12 input vectors, one input vector per data frame. Since two dataset files per bearing class were used in the study, there are 24 input vectors that were prepared per 
bearing class. Therefore, there were 96 feature vectors in total for the four classes of bearing status considered in this study.

Low pass filter was used to eliminate the noise which usually exists in the higher frequency band of the signals. Since the frequencies of interest i.e. the characteristic frequencies are low frequency which are less than $300 \mathrm{~Hz}$ a Butterworth low pass filter with a cut off frequency of $300 \mathrm{~Hz}$ was used.

In order to compute the frequency components of the input vectors, Fast Fourier Transform (FFT) was used to convert time domain data into frequency spectrum and followed by power spectral density (PSD) calculation for the spectrum range. The amplitude for frequency of interest was then obtained from the PSD. To compensate for energy leakage during generating the signatures, a frequency band of $5 \mathrm{~Hz}$ was used when calculating amplitude of characteristic frequencies.

Matlab in-built FFT was used for frequency spectrum generation. The mathematical formula for discrete frequency transform (DFT) is given by,

$$
X(k)=\sum_{n=0}^{N-1} x(n) e^{-j 2 \pi k n / N}, k=0,1,2, \ldots, N-1
$$

Where $x(n)$ represents the time domain data, and $N$ is the number of data point in a data frame.

The following basic formula is converted to Matlab code for PSD calculation.

$$
\begin{gathered}
\operatorname{PSD}(0)=|X(0)|^{2} \\
P S D(k)=|X(k)|^{2}+|X(N-k)|^{2}, k=1,2, \ldots, \frac{N}{2}-1 \\
P S D\left(\frac{N}{2}\right)=\left|X\left(\frac{N}{2}\right)\right|^{2}
\end{gathered}
$$

And the characteristic frequency amplitude calculation formula used within the frequency band of $5 \mathrm{~Hz}$ is,

$$
A\left(F_{c h}\right)=\sum_{F_{c h}-2.5}^{F_{c h}+2.5} P S D\left(\frac{F_{c h}}{\Delta f}\right)
$$

where $\Delta f$ represents the frequency resolution, and $F c h$ is the characteristic frequency.

The characteristic frequencies are calculated using bearing physical parameters obtained for the drive-end bearing of the test rig used by [36] during data acquisition and shown in Table 1.
Table 1. Bearing information for 6205-2RS JEM SKF

\begin{tabular}{|c|c|}
\hline Inside diameter & $0.9843 "$ \\
\hline Outside diameter & $2.0472 "$ \\
\hline Thickness & $0.5906 "$ \\
\hline Ball diameter $(\mathrm{Db})$ & $0.3126^{\prime}$ \\
\hline Cage diameter (Dc) & $1.537^{\prime}$ \\
\hline No. of balls (NB) & 9 \\
\hline Motor speed & $1750 \mathrm{rpm}$ \\
\hline Contact angle, $\Theta$ & $0^{0}$ \\
\hline
\end{tabular}

The frequencies were calculated as follows, Shaft frequency,

$$
F_{S}=\frac{1750}{60}=29.2 \mathrm{~Hz}
$$

Fundamental cage frequency,

$$
F_{C}=\frac{1}{2} F_{S}\left(1-\frac{D_{b} \cos \theta}{D_{c}}\right)=11.6 H z
$$

Ball pass outer race way frequency,

$$
F_{B P O}=\frac{N_{B}}{2} F_{S}\left(1-\frac{D_{b} \cos \theta}{D_{c}}\right)=104.6 \mathrm{~Hz}
$$

Ball pass inner race way frequency,

$$
F_{B P I}=\frac{N_{B}}{2} F_{S}\left(1+\frac{D_{b} \cos \theta}{D_{c}}\right)=158.1 \mathrm{~Hz}
$$

Ball frequency,

$$
F_{B}=\frac{D_{c}}{2 D_{b}} F_{S}\left(1-\frac{D_{b}^{2} \cos ^{2} \theta}{D_{c}^{2}}\right)=137.6 H z
$$

For computation of peak value, mean value and kurtosis of the time domain values, in-built Matlab functions are used. Below are the equations used in statistical computations.

$$
\begin{gathered}
A_{\max }=\max _{n=0}^{N-1}(x(n)) \\
A_{\text {mean }}=\frac{1}{N} \sum_{n=0}^{N-1} x(n) \\
K=\frac{\frac{1}{N} \sum_{n=0}^{N-1}(x(n))^{4}}{\sqrt{\frac{1}{N} \sum_{n=0}^{N-1}(x(n))^{2}}}
\end{gathered}
$$


Using the sample data frames prepared, there were 96 feature vectors each containing 7 elements which form a $96 \times 7$ matrix. This matrix was used as input matrix for the ANN training and it is called feature matrix. A corresponding target output values were also prepared for the training. This target matrix was a 4 x 96 matrix, where the 4 refers to the four bearing classes and 96 refers to the number of training samples. Since the dataset available was for single defect bearing only, multiple defect bearing diagnosis was not considered in this work. Accordingly, the expected bearing status evaluation results are as follows.

$\begin{array}{cc}\text { Target vector for outer raceway fault (OF) is: } & {\left[\begin{array}{l}1 \\ 0 \\ 0 \\ 0\end{array}\right]} \\ \text { Target vector for inner raceway fault (IF) is: } & {\left[\begin{array}{l}0 \\ 1 \\ 0 \\ 0\end{array}\right]} \\ \text { Target vector for ball fault (BF) is: } & {\left[\begin{array}{l}0 \\ 0 \\ 1 \\ 0\end{array}\right]} \\ \text { Target vector for normal bearing (NB) is: } & {\left[\begin{array}{l}0 \\ 0 \\ 0 \\ 1\end{array}\right]}\end{array}$

The feature values were calculated as normalized double precision floating point real numbers using Matlab software.

\section{Dataset manipulation for time domain feature extraction approach}

The time domain feature extraction approach uses statistical parameters of bearing data as input features. It involves less computational task and straight forward interpretations of the results as compared to frequency domain approach [15]. While it is difficult to detect general roughness bearing defects using frequency domain method, the time domain approach is able to detect such faults as the severity level increases. This is because of the change in vibration amplitude when severity increases. Frequency domain approach is more effective in detection of localized defects [5, 34]. The use of time-frequency approach could be the best approach in bearing fault detection. However, this increases computational tasks and the number of input features used during training. In this study, programmable logic controller (PLC) is considered for the implementation phase. PLCs are not made to handle complex mathematical computations which put a limit on the frequency domain feature extraction method. Therefore, the option of using time domain approach only is more feasible.

The time domain features considered were root mean square (RMS) value, variance $(\sigma 2)$, kurtosis and normal negative $\log$ likelihood $(\bar{\Lambda})$ value. These features are reported to give best performance in researches which considered time domain only feature extraction approach of bearing diagnosis [15, 37].

Below are the equations used to compute the time domain features,

$$
\begin{gathered}
R M S=\sqrt{\frac{1}{N} \sum_{n=0}^{N-1}(x(n))^{2}} \\
\sigma^{2}=\frac{1}{N} \sum_{n=0}^{N-1}(x(n)-\mu)^{2} \\
K=\frac{\frac{1}{N} \sum_{n=0}^{N-1}(x(n))^{4}}{\sqrt{\frac{1}{N} \sum_{n=0}^{N-1}(x(n))^{2}}} \\
\Lambda=-\sum_{n=0}^{N-1} \log \left[f\left(x_{i} ; \mu, \sigma\right)\right]
\end{gathered}
$$

where $R M S$ represents the Root Means Square, $\sigma^{2}$ is the variance, $\mu$ is the mean, $K$ is the kurtosis, $\Lambda$ is the normal negative $\log$ likelihood, and $f(x i ; \mu, \sigma)$ is the normal probability density function (pdf) [37].

$$
f\left(x_{i} ; \mu, \sigma\right)=\frac{1}{\sigma \sqrt{2 \pi}} e^{\frac{-\left(x_{i}-\mu\right)^{2}}{2 \sigma^{2}}}
$$

The data manipulation required in time domain is minimal when compared to the frequency domain approach. The original dataset files were segmented directly into 12 data frames containing 5,000 data points. Here 5,000 data points per data frame were tested to be sufficient instead of 10,000 data points used in frequency domain.

The time domain feature vector contains four elements,

$$
\mathrm{X}=\left[\begin{array}{llll}
\mathrm{RMS} & \sigma 2 & \mathrm{~K} & \Lambda
\end{array}\right]
$$

Therefore, the feature matrix with 96 input vectors is $96 \times 4$ while the corresponding target matrix size remains the same for both approaches i.e. 4 x 96. Since less number of data points is required in time domain approach, it was possible to increase further the number of input vectors (samples). But to make the comparison suitable, the sample size was preferred to remain equal.

\section{Neural Network training and performance evaluation}

\section{Neural Network training algorithm}

Once the feature matrix and target matrix are ready, they can be presented to the ANN for training and validation. A three layer feedforward backpropagation neural network with 10 neurons in the hidden layer was created and trained using Matlab software. There were 4 neurons in the output layer where each neuron corresponds to the probability of one class. 


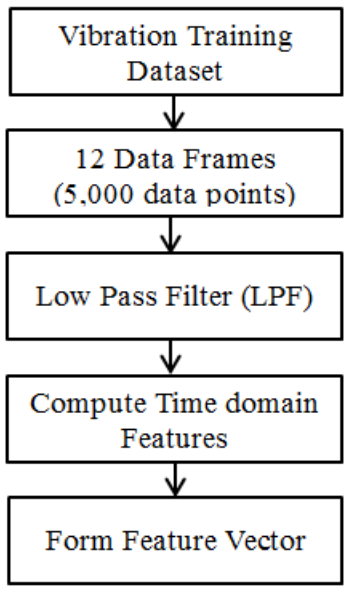

Fig.5. Steps to compute feature vectors in the time domain

The training and validation algorithm used in the study was the Levenberg-Marquardt (LM) training algorithm with the following default settings.

Table 2. Default parameter setting for Levenberg-Marquardt algorithm

\begin{tabular}{|c|c|}
\hline $\begin{array}{c}\text { Maximum number of epochs (iterations) } \\
\text { for training }\end{array}$ & 1000 \\
\hline Performance or error goal & 0 \\
\hline Maximum validation fails & 6 \\
\hline Minimum performance gradient & $1 \times 10^{-7}$ \\
\hline Initial learning rate, $\mu$ & 0.001 \\
\hline$\mu$ decrease factor & 0.1 \\
\hline$\mu$ increase factor & 10 \\
\hline Maximum value for $\mu$ & $1 \times 10^{10}$ \\
\hline Maximum training time $(\mathrm{sec})$ & $\inf$ \\
\hline
\end{tabular}

The feedforward neural network training tool of Matlab uses sigmoid activation functions for hidden neurons and linear activation functions for output neurons as default settings. The same setting was used in this study.

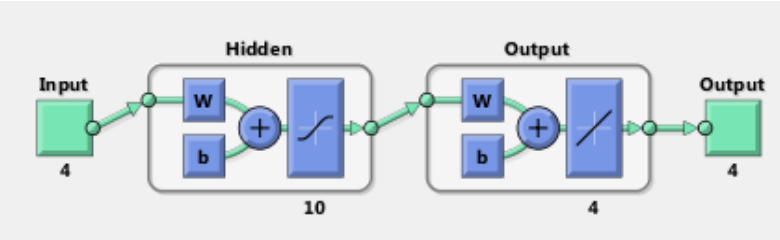

Fig.6. Feedforward neural network created using Matlab neural network training tool

Once training of the neural network is started, it stops when one of the following conditions occur:

a) The maximum number of epochs is reached

b) The maximum training time is exceeded

c) Performance goal is achieved

d) Performance gradient falls below minimum setting

e) Learning rate exceeds maximum setting

f) Maximum number of validation fails is exceeded

Neural network training can be repeated until best performance is obtained. The trained neural network is then saved for further test with additional datasets or for deploying into the PLC. Increasing the number of hidden neurons beyond 10 did not improve the results.

\section{Performance evaluation using test datasets}

The performance of trained neural network can be further tested using separate dataset files. A different dataset file for each bearing class was downloaded from [36] as a test dataset. Similar dataset manipulation steps were taken to prepare feature vectors for the testing purpose as shown in Fig. 2. (b) above. Twelve input vectors were prepared for each bearing class for the testing purpose and the outputs were recorded. The detection rate observed was $100 \%$ for all bearing classes within the test. Further information is presented under Section 4 below.

\section{Tests on important variables}

\section{Test on effect of number of input features}

The nature and number of input features selected for ANN training is an important factor. For time-frequency domain approach, before choosing the seven input features described under Section 3.B above, amplitude of ball frequency was considered as the eighth input and the detection result remained $100 \%$. But it was unnecessary to include the eighth feature since the detection rate was already $100 \%$ with the seven feature inputs. And also the shaft frequency was removed and tests were conducted with six inputs only. The detection result was nearly $100 \%$ with slightly more performance error especially on outer race way and inner race way faults. The detection error is expected to increase with varying motor speed where average speed should be used for training input. This feature is considered as important since the motor speed usually varies when operating under different load levels.

For time domain approach, root mean square and variance values alone were tested for performance. The overall success rate was only $89 \%$. Kurtosis was used along with normal negative log likelihood for performance test. The detection rate was $100 \%$ in detecting ball faults and normal bearings but it performed poor in detection of outer race faults and inner race faults. The best performance was obtained when four of them were used together. Skewness (S) was used as a fifth input feature but no performance improvement was observed.

Table 3. Number of features and ANN training performance

\begin{tabular}{|c|c|c|}
\hline $\begin{array}{l}\text { Types of } \\
\text { Features }\end{array}$ & Features involved during the test & $\begin{array}{c}\text { Performance } \\
\text { (accuracy) }\end{array}$ \\
\hline \multirow{3}{*}{$\begin{array}{c}\text { Time- } \\
\text { frequency } \\
\text { domain } \\
\text { features }\end{array}$} & $A_{\max } A_{\text {mean }} K F_{S} \quad A_{S} \quad A_{O} \quad A_{I} \quad A_{B}$ & $100 \%$ \\
\hline & $A_{\max } A_{\text {mean }} K F_{S} \quad A_{S} \quad A_{O} A_{I}$ & $100 \%$ \\
\hline & $A_{\max } A_{\text {mean }} K \quad A_{S} \quad A_{O} \quad A_{I}$ & $99.99 \%$ \\
\hline \multirow{4}{*}{$\begin{array}{c}\text { Time } \\
\text { domain } \\
\text { features }\end{array}$} & RMS $\sigma^{2}$ & $89 \%$ \\
\hline & $\mathrm{K} \Lambda$ & $78 \%$ \\
\hline & $\mathrm{RMS} \sigma^{2} \mathrm{~K} \Lambda$ & $100 \%$ \\
\hline & $\mathrm{RMS} \sigma^{2} \mathrm{~K} \Lambda \mathrm{S}$ & $100 \%$ \\
\hline
\end{tabular}




\section{Test on effect of number of data points}

Optimum number of data points should be used for a good performance. In frequency domain, the frequency resolution and the energy of frequency spectrum decreases as the number of data points decrease. Below is a plot of frequency spectrum using dataset from outer race fault bearing for illustration of effect of number of data points.

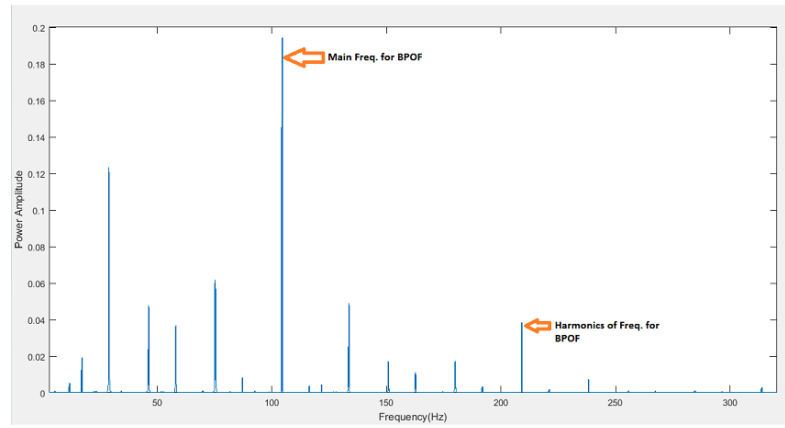

(a) Plot with 120,000 data points

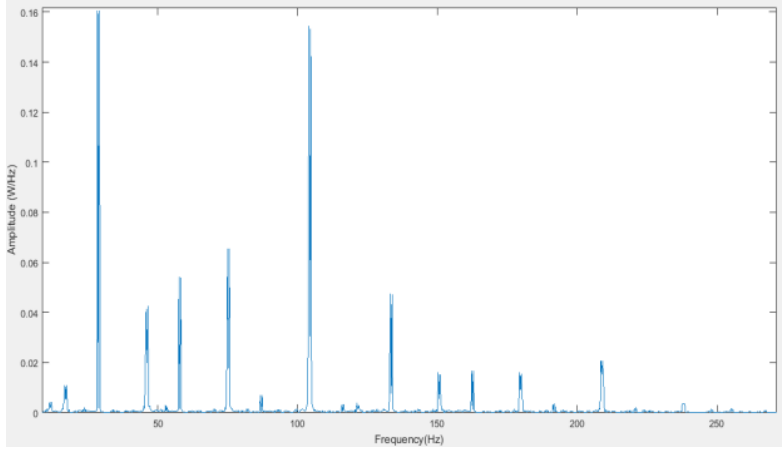

(b) Plot with 10,000 data points

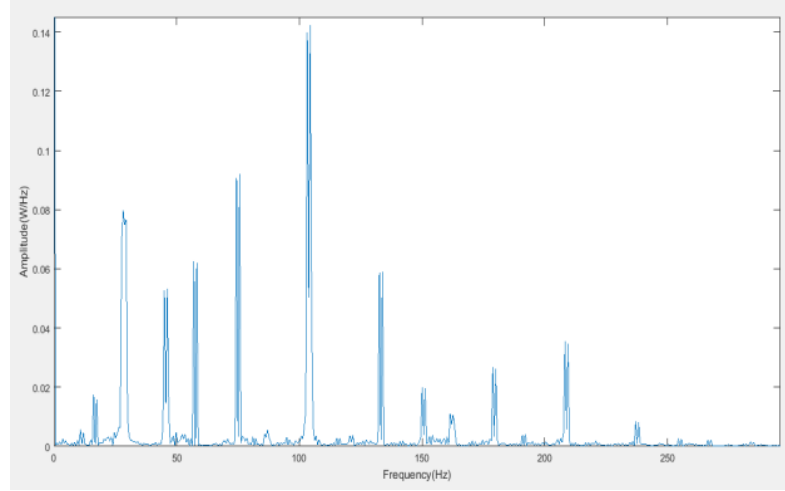

(c) Plot with 5,000 data points

Fig.7. Effect of number of data points on frequency resolution and energy of spectrum

It can be observed from Fig. 7. (c) above that the frequency resolution is highly affected with 5,000 data points. It is also observed that the amplitude (energy) of the spectrum decreases as the number of data points decrease.

Similar test was done for the time domain approach and optimally high performance result for all bearing classes was obtained at 5,000 data points. The test using 2,000 data points resulted in low detection rate for inner race fault with accuracy of $77.5 \%$. A detection accuracy of $100 \%$ was recorded for the other three classes.

\section{Test on effect of noise and the use of low pass filter}

Additive White Gaussian Noise (AWGN) was used to test the effect of noise on the results of detection. Both frequency domain and time domain approaches were affected by additive noise but the frequency domain was found to be more sensitive to noises. Pairs of frequency domain plots are shown below where one of the plots include additive noise with signal to noise ratio (SNR) value of 10 and the other after applying Butterworth low pass filter to the signal affected by noise. The Butterworth cut-off frequency used was $300 \mathrm{~Hz}$.

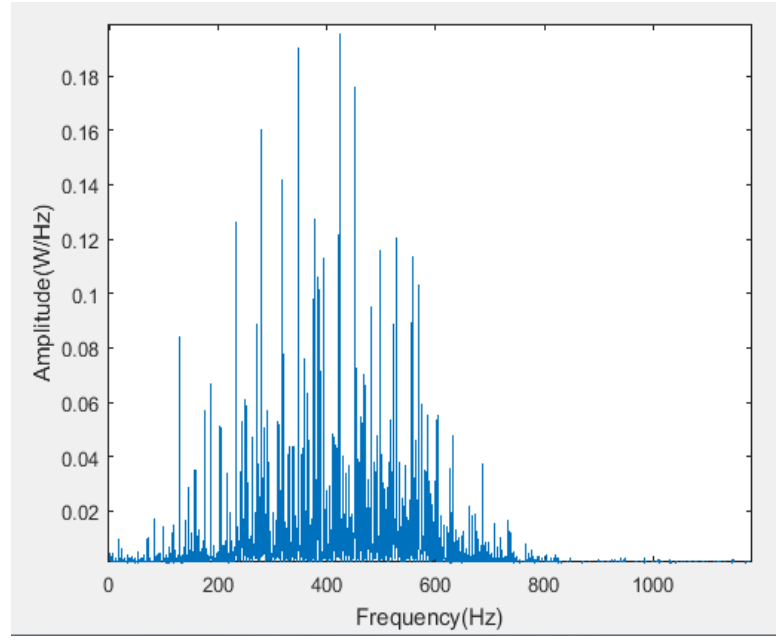

Fig.8. Effect of white noise, SNR of 10, on frequency spectrum

The effect of noise was avoided by using Butterworth low pass filter as observed from Fig. 9.

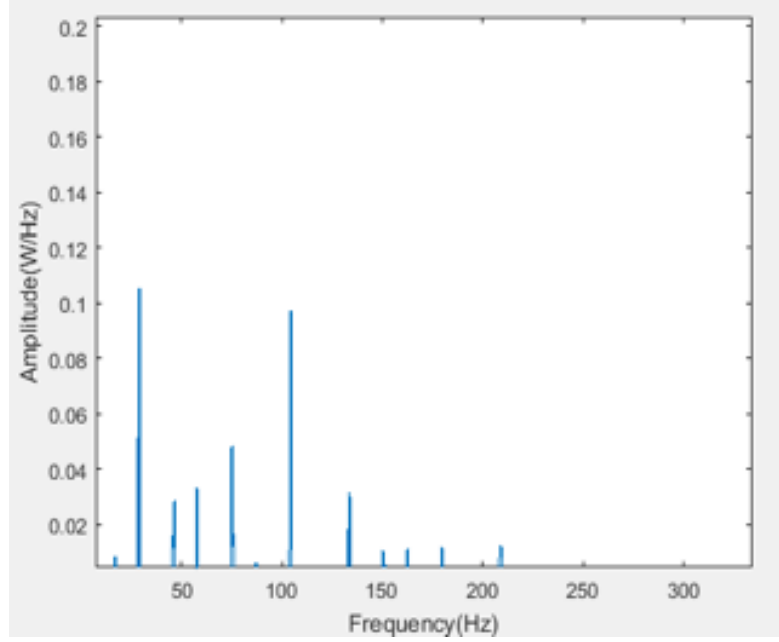

Fig.9. Effect of white noise, SNR of 10, on frequency spectrum after applying a Butterworth filter

The table below gives detection accuracy results obtained by adding white noise to test datasets at different SNR values. 
Table 4. ANN detection accuracy with white noise

\begin{tabular}{|c|c|c|c|}
\hline $\begin{array}{c}\text { Test } \\
\text { No. }\end{array}$ & SNR & $\begin{array}{c}\text { Detection accuracy } \\
\text { with time-frequency } \\
\text { features }\end{array}$ & $\begin{array}{c}\text { Detection accuracy } \\
\text { with time domain } \\
\text { features }\end{array}$ \\
\hline 1 & 5 & $86.4 \%$ & $98.7 \%$ \\
\hline 2 & 10 & $92.9 \%$ & $100 \%$ \\
\hline 3 & 20 & $93.6 \%$ & $100 \%$ \\
\hline
\end{tabular}

It was observed during the test that the detection accuracy of time-frequency approach did not show significant improvement for SNR values of greater than 20. It is clear from the data that time domain features were not affected by noise for SNR values of greater than 10. This is one of the advantages of using time domain approach.

\section{RESULTS AND DISCUSSION}

This section deals with key results obtained during experimentation in simulation and implementation phases. The results obtained were based on the methodology discussed in Section 3.

\section{A. Frequency domain feature extraction}

The following results were obtained using 10,000 data points to compute input features. The indication of a specific bearing defect is the presence of its associated characteristic frequency within the frequency spectrum. The magnitude of the amplitudes observed is proportional to the impact level created due to the defect during bearing rotation. It also depends on the position of accelerometer during data recording. We can observe from Fig. 10. below that the impact level decreases as we go from outer race fault to inner race fault and then to ball fault. For higher defect levels or severity levels, we can see the second harmonics in addition to the primary characteristic frequency. Second harmonics is 2 times the first harmonic by definition [10].

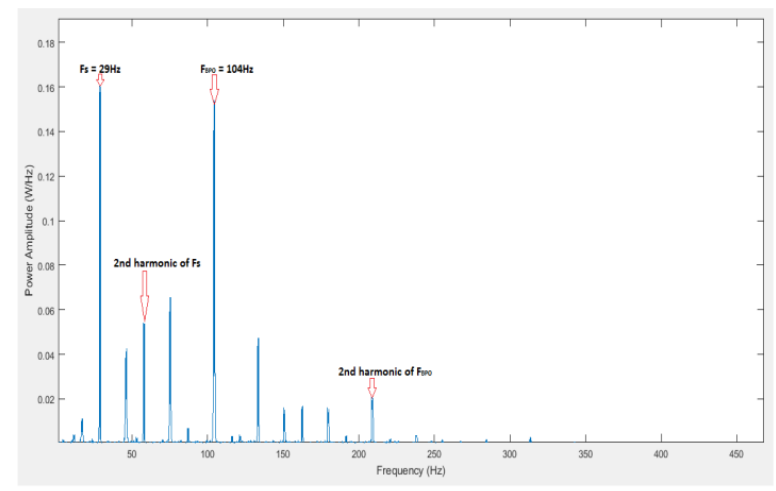

(a) Outer raceway fault frequency spectrum

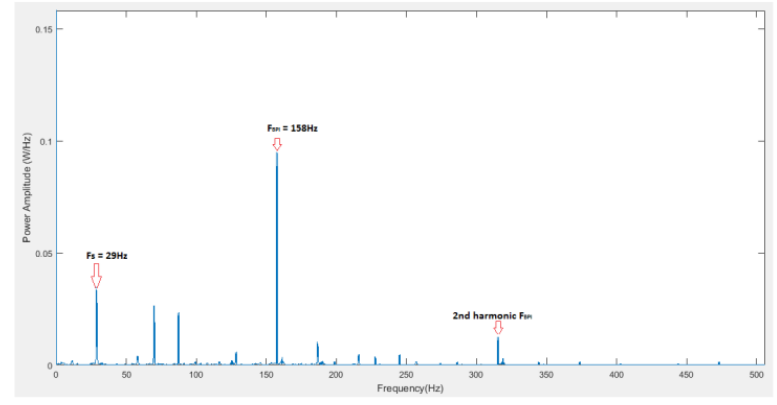

(b) Inner raceway fault frequency spectrum

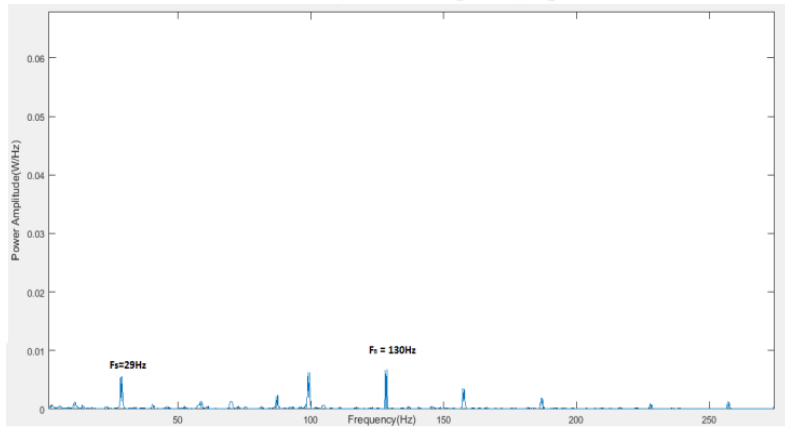

(c) Ball fault frequency spectrum

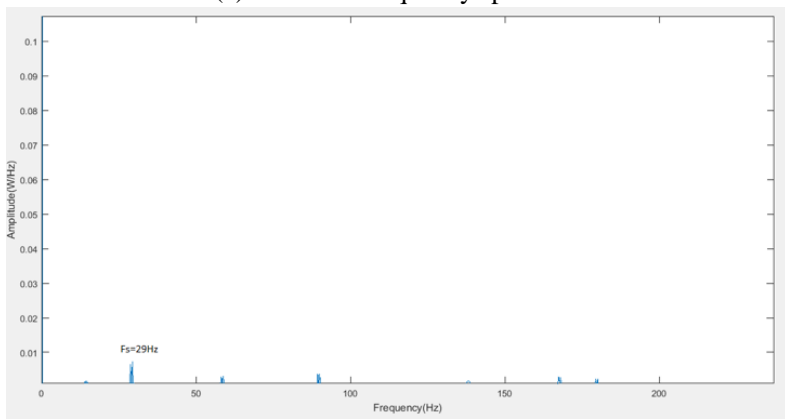

(d) Normal bearing frequency spectrum

Fig.10. Frequency spectrums for (a) outer race fault (b) inner race fault (c) ball fault (d) normal

As seen in Fig.10. above, some unknown frequencies are also available in the spectrum which could be due to various vibrations in the motor system or the surrounding which are not related to bearing faults. The shaft frequency is available in all bearing class spectrums which is expected as long as the motor is running.

The required features are clearly observed for all bearing classes. The characteristic frequency amplitudes within $\pm 2.5 \mathrm{~Hz}$ bandwidth were used to form the feature vectors to compensate for energy leakage during spectrum generation. There was only a slight drift of the frequency values as compared to the calculated values except the ball fault characteristic frequency. The calculated frequency for ball fault is $137.6 \mathrm{~Hz}$ but the value obtained during simulation was $130 \mathrm{~Hz}$. The difference is about $7.6 \mathrm{~Hz}$ which lies outside the compensation bandwidth. However, ball fault amplitude is not a selected feature for ANN training as described in Section 3 and does not affect the result. 
The normal bearing frequency spectrum did not show any significant frequency other than the shaft frequency as expected.

\section{B. Time domain feature extraction}

The time domain feature extraction is a simple computation of selected statistical values. Here 5,000 data points per feature vector were used to calculate root mean square, variance, kurtosis and normal negative log likelihood values. Below are the vibration waveforms for the four bearing classes.

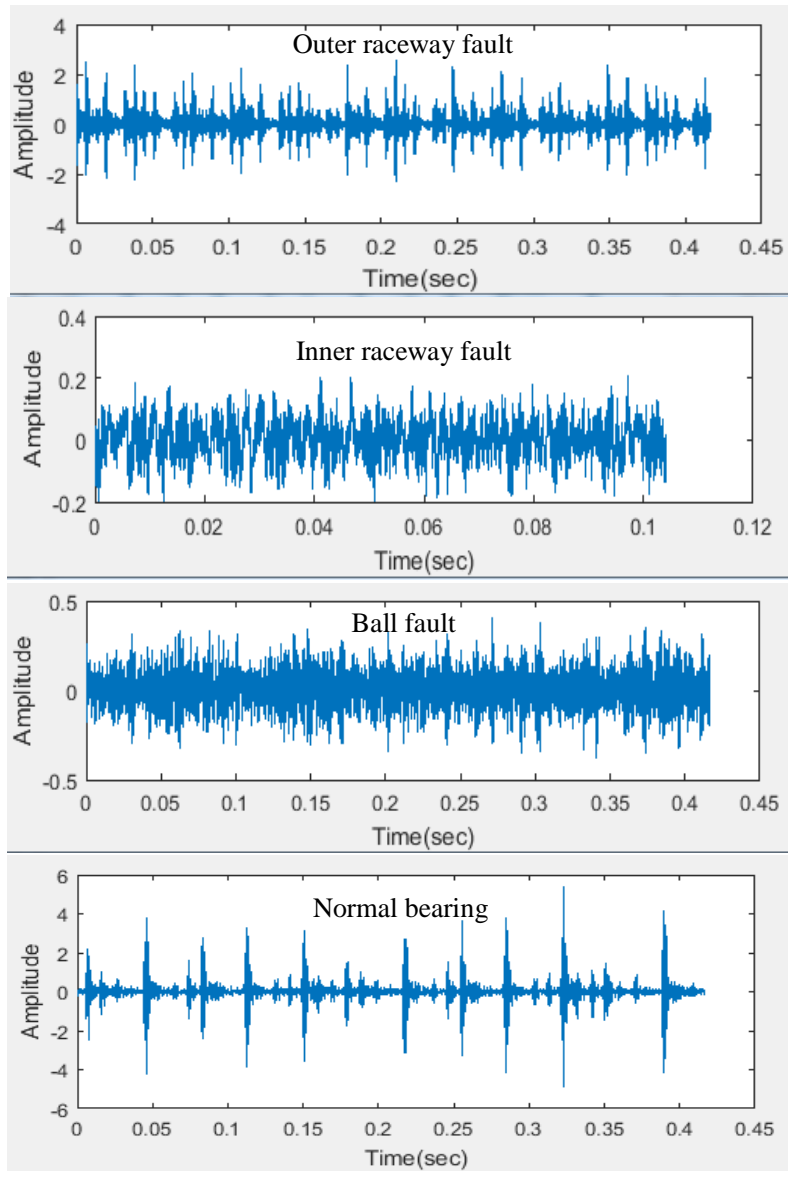

Fig.11. Time domain vibration waveforms

The waveforms show that there are different patterns for each bearing class. The neural network can learn these patterns and based on the training it is able to detect similar patterns during testing and online implementation. The time domain input features were calculated for each bearing class for training.

\section{Neural Network training, validation and testing} results

The results of training and validation of the neural network using Levenberg-Marquardt (LM) training algorithm for both time-frequency domain approach and time domain only approach is provided below.

\section{Training results}

Table 5. Training parameters for time-frequency and time domain features

\begin{tabular}{|c|c|c|c|c|}
\hline & $\begin{array}{c}\text { Average } \\
\text { performance }\end{array}$ & $\begin{array}{c}\text { Average no. } \\
\text { of epochs }\end{array}$ & $\begin{array}{c}\text { Average } \\
\text { gradient } \\
\text { value } \\
\text { reached }\end{array}$ & $\begin{array}{c}\text { Average } \\
\text { learning rate }\end{array}$ \\
\hline $\begin{array}{c}\text { Time- } \\
\text { frequency } \\
\text { domain }\end{array}$ & $1.2 \times 10^{-12}$ & 20 & $3.03 \times 10^{-8}$ & $1.0 \times 10^{-14}$ \\
\hline Time domain & $3.63 \times 10^{-10}$ & 25 & $8.6 \times 10^{-8}$ & $1.25 \times 10^{-10}$ \\
\hline
\end{tabular}

\section{10-fold cross validation performance results}

The performance of the neural network can be further evaluated using 10-fold performance estimation method. The training set of 96 samples was divided into training sets and validation sets for each round of training. In six of the rounds, 86 training sets and 10 validation sets were used. For the rest of four rounds, 87 training sets and 9 validation sets were used.

The NN model used in this study was a three layer feedforward backpropagation network with 10 neurons in the hidden layer. The calculated average performance values were $2.1 \times 10^{-6}$ and $7.9 \times 10^{-4}$ for time-frequency domain and time domain approaches respectively. Thus, the 10-fold cross validation result shows that timefrequency domain feature extraction method has a better performance as compared to time domain technique. The number of hidden neurons was increased to 20 for comparison purpose but there was no performance improvement observed.

\section{Performance test results with test datasets}

Apart from validation check during training and 10fold cross validation performance test described above, additional performance test was conducted using separate dataset files prepared for testing purposes. Separate datasets which involved different defect severity levels were used during the performance test. The test was done for all bearing classes with 12 samples each and with both feature extraction techniques. The test datasets used in both techniques was the same for respective bearing class. The test result is presented in below tables.

Table 6. Time-frequency approach test results with test datasets

\begin{tabular}{|c|c|c|}
\hline Test & Test Result & $\begin{array}{l}\text { Performan } \\
\text { ce (Error) }\end{array}$ \\
\hline $\begin{array}{l}\text { Outer } \\
\text { race fault }\end{array}$ & 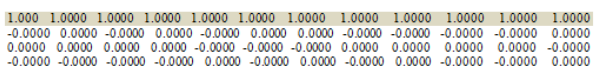 & $2.8 \times 10^{-11}$ \\
\hline $\begin{array}{l}\text { Inner } \\
\text { race fault }\end{array}$ & 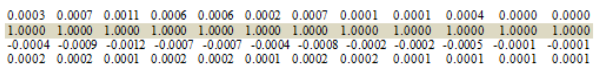 & $1.6 \times 10^{-077}$ \\
\hline Ball fault & 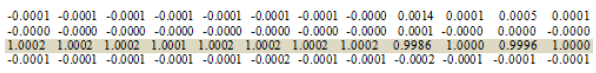 & $9.6 \times 10^{-08}$ \\
\hline $\begin{array}{l}\text { Nomal } \\
\text { bearing }\end{array}$ & 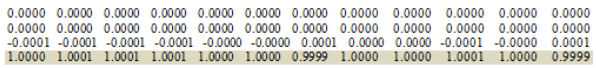 & $2.2 \times 10^{-09}$ \\
\hline
\end{tabular}


Table 7. Time domain approach test results with test datasets

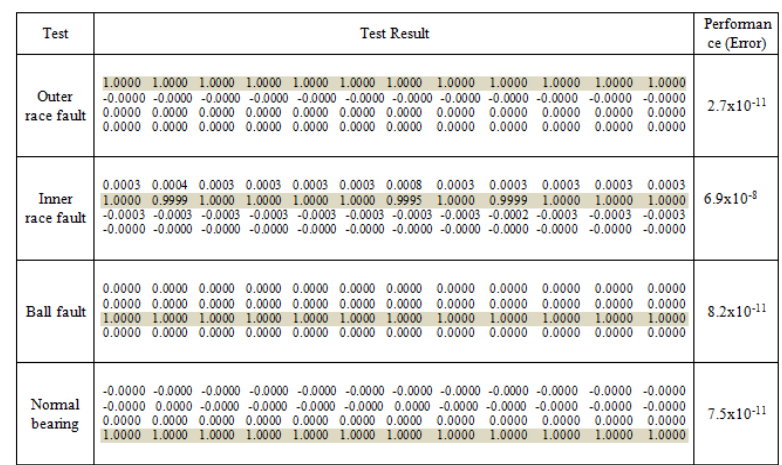

The classification output of both approaches showed a very good performance with test datasets for all bearing classes. Unlike training and validation performance results, the test conducted with test dataset has shown that the performance of time-frequency domain and time domain techniques are nearly the same with average errors of $6.46 \times 10^{-8}$ and $1.73 \times 10^{-8}$ respectively.

\section{Neural Network PLC implementation results}

A neural network that has been trained, validated and tested on Matlab software was deployed into PLC for implementation level tests. The tests were conducted using S7-PLCSIM soft PLC from Siemens. It is a standard simulator used by Siemens to test and debug PLC projects before site installation and commissioning [3].
Feature extraction and bearing diagnosis neural network function blocks (FB) were developed in S7-SCL language. The test dataset prepared on Matlab was imported to the PLC for implementation tests. These test datasets are different from training dataset but the same as the test datasets mentioned in Section 4.C.3 above.

Implementation tests were conducted for both feature extraction techniques. It was observed that performance of a PLC is poor in handling the frequency features. This is mainly due to the time domain to frequency domain conversion algorithm involved during feature extraction. Fast Fourier Transform (FFT) is the most common algorithm for the conversion but it requires high CPU scan time and consumes high memory area as a result of the loop instructions and sine/cosine functions involved.

Another drawback of handling frequency domain features in the PLC is the higher number of data points required for feature vectors as compared to time domain. As mentioned in Section 3, optimum number of data points required was 10,000 i.e. 10,000 floating point real numbers each of them occupying 4 bytes of memory just to import and store. This is roughly $40 \mathrm{~KB}$ of data. Moreover, there is large number of intermediate data that needs to be stored temporarily during mathematical computations by FFT and PSD algorithms. The intermediate values take more memory since they are stored as real and imaginary values for a single complex number during conversion into frequency domain. This may occupy 4 - 5 times of memory area required to store imported data.

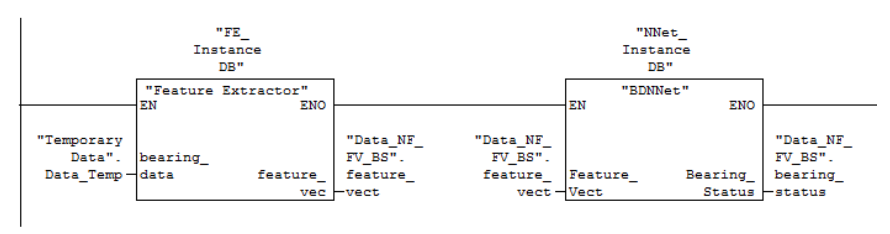

Fig.12. Feature extraction and bearing diagnosis NN function blocks in time domain approach

The time domain feature extraction involves simple arithmetic operations and mathematical functions with short instruction execution time. The results of CPU scan cycle time for both approaches are as follows:

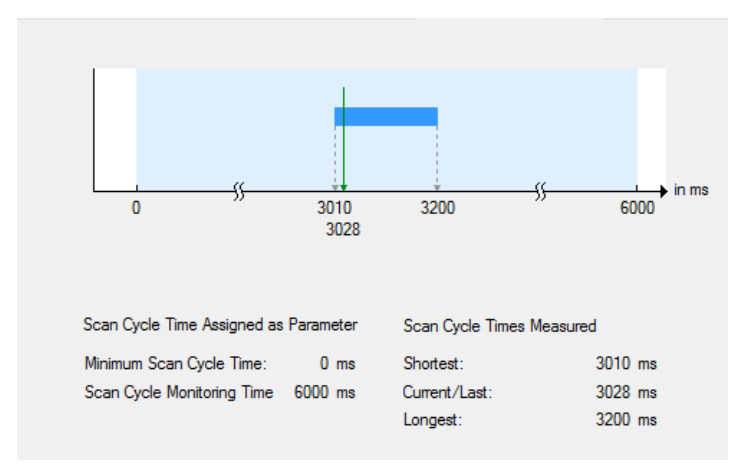

Fig.13. CPU scan cycle time for time-frequency approach

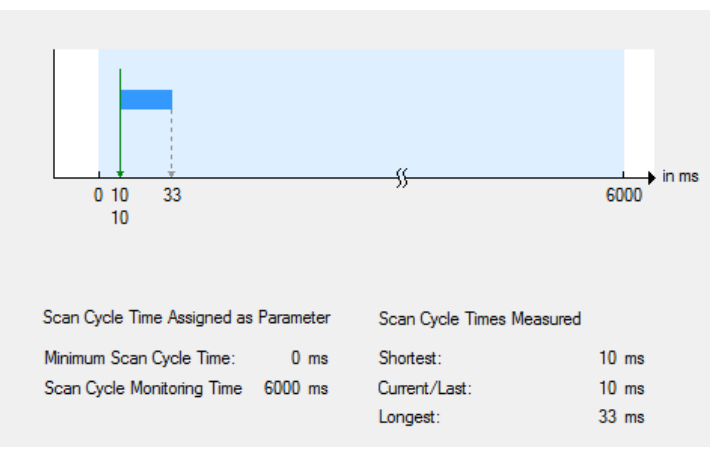

Fig.14. CPU scan cycle time for time domain approach

The results of scan cycle time indicate that time domain approach requires about 10 milliseconds of time whereas the time-frequency approach lasts for about 3 seconds. This shows the time domain approach can be adapted for real-time applications whereas the time- 
frequency approach is not acceptable for real-time applications. This statement assumes the bearing diagnosis application runs in addition to other control tasks that require fast scan cycle time which is usually less than 50ms [19]. The summary of advantages and disadvantages of both approaches with regard to neural network bearing diagnosis using PLCs is presented in the following table.

Table 8. Comparison of time-frequency and time domain feature extraction methods

\begin{tabular}{|c|c|c|}
\hline $\begin{array}{c}\text { Feature } \\
\text { Extraction } \\
\text { Method }\end{array}$ & Advantages & Disadvantages \\
\hline $\begin{array}{l}\text { Time- } \\
\text { frequency } \\
\text { domain } \\
\text { approach }\end{array}$ & $\begin{array}{c}\text { More accurate in } \\
\text { detecting localized } \\
\text { bearing faults } \\
\text { Higher performance } \\
\text { during NN training and } \\
\text { validation } \\
\text { Less number of epochs } \\
\text { during training }\end{array}$ & $\begin{array}{c}\text { Difficult to detect } \\
\text { general roughness } \\
\text { faults } \\
\text { More sensitive to } \\
\text { noises } \\
\text { Requires higher } \\
\text { number of data points } \\
\text { and higher number of } \\
\text { input features. } \\
\text { Requires higher CPU } \\
\text { scan cycle time and } \\
\text { higher memory area }\end{array}$ \\
\hline $\begin{array}{c}\text { Time domain } \\
\text { approach }\end{array}$ & $\begin{array}{c}\text { Involves simple } \\
\text { statistical values as input } \\
\text { features } \\
\text { Can detect both } \\
\text { localized defects and } \\
\text { general roughness faults } \\
\text { Requires less number of } \\
\text { data points and less } \\
\text { number of input features } \\
\text { Easy to adapt to PLC } \\
\text { program since it does } \\
\text { not involve complex } \\
\text { algorithm. } \\
\text { Requires lower CPU } \\
\text { scan cycle time and low } \\
\text { memory area } \\
\text { Less sensitive to noises }\end{array}$ & $\begin{array}{l}\text { Relatively less } \\
\text { accurate in detecting } \\
\text { very small defects. } \\
\text { Lower performance } \\
\text { during training and } \\
\text { validation } \\
\text { Requires more } \\
\text { number of epochs to } \\
\text { reach minimum } \\
\text { gradient }\end{array}$ \\
\hline
\end{tabular}

In view of above comparison, time domain is the best approach for PLC implementation.

\section{CONCLUSION}

The main target of this work is to test the feasibility of Artificial Neural Network (ANN) implementation on a standard Programmable Logic Controller (PLC) for motor bearing diagnosis applications. The research was conducted on bearing vibration dataset obtained from Case Western Reserve University (CWRU) data center. The data was recorded for normal bearing, bearing with outer raceway fault, bearing with inner raceway fault and bearing with ball fault.

A three layer multilayer perceptron (MLP) backpropagation neural network was proposed as a classifier. Time-frequency domain and time domain only features were investigated. Both features have shown good detection performance with an average performance error of $2.1 \times 10^{-6}$ for time-frequency approach and 7.9 $\mathrm{x} 10^{-4}$ for the time domain approach.

A PLC implementation simulation was performed using Siemens SIMATIC S7-300/400 Software and S7PLCSIM PLC simulator. The hardware implementation simulation showed that the use of time domain features is better suited for real-time applications than timefrequency domain ones. The scan cycle time obtained was about 3 seconds for time-frequency features, which is not suitable for real-time applications where the same PLC is typically used for general control tasks and bearing diagnosis. The scan cycle time obtained with time domain features is only $10 \mathrm{~ms}$ which is one of the shortest scan cycle times in PLCs.

Therefore, a PLC implementation of an ANN-based bearing diagnosis is proved as feasible with the use of time domain features.

As compared to other hardware implementations of ANN-based bearing diagnosis such Very Large Scale Integration (VLSI) and Field Programmable Gate Arrays (FPGA), PLC implementation is the cheapest approach wherever the bearing diagnosis blocks can be included to a general purpose PLC. Unlike the others, the core solution in PLC implementation approach is software based which guarantees its flexibility. The only hardware required is an accelerometer connected to the PLC for vibration data collection. Moreover, PLCs are prevalent industrial controllers that have been in use for almost 50 years with a proven performance. This gives a wide opportunity for implementation of the bearing diagnosis on existing installations as well as new projects.

\section{ACKNOWLEDGMENTS}

The authors would like to thank Jehoiachin Techno for sponsoring this study, the Case Western Reserve University (CWRU) for providing the bearing datasets used in this research, and the anonymous reviewers for their constructive advice.

\section{REFERENCES}

[1] R. D. Abdu-Aljabar, "Design and Implementation of Neural Network in FPGA," Journal of Engineering and Development, vol. 16, no. 3, September 2012.

[2] J. Lehtonen and H. Koivo, "Fault Diagnosis of Induction Motors with Dynamical Neural Networks," in IEEE International Conference on Systems, Man and Cybernetics, 2005, pp. 2979 - 2984.

[3] W.Bolton, Programmable Logic Controllers, 5th ed. Burlington, USA: Newnes, 2009.

[4] M. Hajar, A. Raad and M. Khalil, "Bearing and Gear Fault Detection Using Artificial Neural Networks," in International Conference on Advances in Computational Tools for Engineering Applications, Zouk Mosbeh, Lebanon, 2013.

[5] L. Wang, "Induction Motor Bearing fault detection Using a Sensorless Approach," Texas A\&M University, College Station, PhD Thesis 2007.

[6] S. Bhattacharyya, D. Sen, S. Adhvaryyu and C. Mukherjee, "Induction Motor Fault Diagnosis by Motor Current Signature Analysis and Neural Network Techniques," Journal of Advanced Computing and Communication Technologies, vol. 3, no. 1, pp. 12-18, February 2015.

[7] X.Z.Gao and S.J. Ovaska, "Soft computing methods in motor fault diagnosis," International Journal of Applied 
Soft Computing, vol. 1, no. 1, pp. 73-81, June 2001.

[8] B. Li, M. Chow, Y.Tipsuwan and J.C.Hung, "NeuralNetwork-Based Motor Rolling Bearing Fault Diagnosis," IEEE Transaction on Industrial Electronics, vol. 47, no. 5, pp. 1060 - 1069, October 2000.

[9] L. Eren, "Bearing Fault Detection by One-Dimensional Convolutional Neural Networks," Mathematical Problems in Engineering, vol. 2017, pp. 1-9, June 2017.

[10] M. Unal, M. Demetgul, M. Onat and H. Kucuk, "Fault Diagnosis of Rolling Bearing Based on Feature Extraction and Neural Network Algorithm," Recent Advances in Electrical Engineering Series, vol. 10, pp. 179 - 185, March 2013.

[11] L. Fausett, Fundamentals of Neural Networks: Architectures, Algorithms and Applications. Upper Saddle River, New Jersey, USA: Prentice-Hall, 1994.

[12] Z. Huo, Y. Zhang, P. Francq, L. Shu and J. Huang, "Incipient Fault Diagnosis of Roller Bearing using Optimized Wavelet Transform based Multi-speed Vibration Signatures," IEEE Access, pp. 1-1, March 2017.

[13] D.H. Pandya, S.H. Upadhyay and S.P. Harsha, "ANN Based Fault Diagnosis of Rolli ng Element Bearing Using Time-Frequency Domain Feature," International Journal of Engineering Science and Technology, vol. 4, no. 6, pp. 2878 - 2886, June 2012.

[14] K. F. Al-Raheem and W.A.Karem, "Rolling bearing fault diagnostics using artificial neural networks based on Laplace wavelet analysis," International Journal of Engineering, Science and Technology, vol. 2, no. 6, pp. $278-290,2010$.

[15] B. Sreejith, A.K. Verma and A. Srividya, "Fault diagnosis of rolling element bearing using time-domain features and neural networks," in IEEE Region 10 Colloquium and the Third ICIIS, Kharagpur, 2008, pp. 1-6.

[16] J. P. Patel and S. H. Upadhyay, "Comparison between Artificial Neural Network and Support Vector Method for a Fault Diagnostics in Rolling Element Bearings," in International Conference on Vibration Problems, Guwahati, 2015, pp. 390 - 397.

[17] A. Nabhan, N. Ghazaly, A. Samy and M. Mousa, "Bearing Fault Detection Techniques - A Review," Turkish Journal of Engineering, Sciences and Technology, vol. 3, pp. 1-18, January 2015.

[18] Y. Xie and T. Zhang, "Fault Diagnosis for Rotating Machinery Based on Convolutional Neural Network and Empirical Mode Decomposition," Shock and Vibration, vol. 2017, pp. 1-12, August 2017.

[19] L. A. Bryan and E.A. Bryan, Programmable Controllers Theory and Implementation, 2nd ed. Marietta, Georgia, USA: Industrial Text Company, 1997.

[20] K.C. D. Kompella, V.G.R. Mannamb and S. R. Rayapudica, "DWT based bearing fault detection in induction motor using noisecancellation," Journal of Electrical Systems and Information Technology, vol. 3, pp. 411 - 427, August 2016.

[21] A.Wadekar and A. Jawadekar, "Fault Detection of Induction Motor through the Analysis of Stator Current Data," International Journal of Industrial Electronics and Electrical Engineering, vol. 5, no. 8, pp. 31 - 35, August 2017.

[22] S. Ergin, A. Uzuntas and M. B.Gulmezoglu, "Detection of Stator, Bearing and Rotor Faults in Induction Motors," in International Conference on Communication Technology and System Design, Coimbatore, 2011, pp. 1103 - 1109.

[23] K.B.Detienne, D.H.Detienne and S.A.Joshi, "Neural Networks as Statistical Tools for Business Researchers," Organizational Research Methods, vol. 6, no. 2, pp. 236 -
265, April 2003.

[24] C.G.Nayak, M. Heichel and S. G Nayak, "Analog Signal in Programmable Logic Controlllers," International Journal of Electrical, Electronics and Data Communication, vol. 3, no. 2, pp. 58 - 61, February 2015.

[25] S. Ray, "Improve Your Model Performance using Cross Validation," Analytics Vidhya, Mar.03, 2018.[Online].Available: https://www.analyticsvidhya.com/blog/2018/05/improvemodel-performance-cross-validation-in-python-r/. [Accessed May 15, 2018].

[26] I.Topalova and A.Tzokev, "Modular Structure of Neural Networks for Classification of Wooden Surfaces with PLC Industrial Implementation," in International Conference on Autonomic and Autonomous Systems, Venice, 2011, pp. 13 - 17.

[27] J.Geiman," On-line Motor Monitoring," Vibration Institute.[Online].

Available:http://www.vibration.org/Presentation/Joe\%20 Geiman\%20-\%20Baker\%20Instruments.pdf [Accessed May 22, 2018]

[28] E. El-Araby, M.A. Bardiny, B. A. Zala and M. I. Mahmoud, "Real-Time Neural Network Speed Controller Implementation on the PLC for a DC Drive," Faculty of Electronic Engineering Periodical, pp. 1 - 8, June 2008.

[29] C. Fairclough, "Analyzing the Structural Integrity of an Induction Motor with Simulation," COMSOL, May 11, 2017.[online]. Available: https://www.comsol.com/blogs/analyzing-the-structuralintegrity-of-an-induction-motor-with-simulation/. [Accessed May 22, 2018].

[30] D. Svozil, V. KvasniEka and J. Pospichal, "Introduction to multi-layer feed-forward neural networks," Chemometrics and Intelligent Laboratory Systems, vol. 39, pp. 43 - 62, June 1997.

[31] J. K. Basu, D. Bhattacharyya and T. Kim, "Use of Artificial Neural Network in Pattern Recognition," International Journal of Software Engineering and Its Applications, vol. 4, no. 2, pp. 23 - 33, April 2010.

[32] A.Thilagavathy and K.V Kanth, "Digital Hardware Implementation of Artificial Neural Network for Signal Processing," International Journal of Scientific \& Engineering Research, vol. 4, no. 3, pp. 1 - 4, March 2013.

[33] A. Dubey, "A Study of Classification Techniques Using Soft Computing," Guru Ghasidas Vishwavidyalaya Bilaspur, Bilaspur, $\mathrm{PhD}$ Thesis 2011.

[34] J. R. Stack, T. G. Habetler and R. G. Harley, "Fault Classification and Fault Signature Production for Rolling Element Bearings in Electric Machines," IEEE Transactions on Industry Applications, vol. 40, no. 3, pp. 735 - 739, June 2004.

[35] X. Wang, Y. Zheng, Z. Zhao and J. Wang, "Bearing Fault Diagnosis Based on Statistical Locally Linear Embedding," Sensors, vol. 15, no. 7, pp. 16225-16247, July 2015.

[36] Case Western Reserve University, "Bearing Data Center Seeded Fault Test Data," Case Western Reserve University Bearing Data Center. [Online]. Available:https://csegroups.case.edu/bearingdatacenter/pa ges/welcome-case-western-reserve-university-bearingdata-center-website

[37] L. Pratyusha, S. Priya and V. Naidu, "Bearing Health Condition Monitoring: Time Domain Analysis," International Journal of Advanced Research in Electrical,Electronics and Instrumentation Engineering, vol. 3, no. 5, pp. 75 - 82, December 2014.

[38] W. Li and C.K.Mechefske, "Detection of Induction Motor 
Faults: A Comparison of Stator Current, Vibration and Acoustic Methods," Journal of Vibration and Control, vol. 12, no. 2, pp. 165 - 188, February 2006.

[39] M. Natu, "Bearing Fault Analysis Using Frequency Analysis and Wavelet Analysis," International Journal of Innovation, Management and Technology, vol. 4, no. 1, pp. 90 - 92, February 2013.

[40] S. Patidar and P.K. Soni, "An Overview on Vibration Analysis Techniques for the Diagnosis of Rolling Element Bearing Faults," International Journal of Engineering Trends and Technology (IJETT), vol. 4, no. 5, pp. 1804 1809, May 2013.

[41] S. Sahin and Y. Becerkli, "Neural Network Implementation in Hardware Using FPGAs," in International Conference on Neural Information Processing (ICONIP), Hong Kong, 2006, pp. 1105 - 1112.

[42] B.D.Yammenavar, V.R.Gurunaik, R.N.Bevinagidad and V.U.Gandage, "Design and Analog VLSI Implementation of Artificial Neural Network," International Journal of Artificial Intelligence \& Applications (IJAIA), vol. 2, no. 3, pp. 96 - 109, July 2011.

[43] N. Cotton, B. Wilamowski and G. Dundar, "A Neural Network Implementation on an Inexpensive Eight Bit Microcontroller," in International Conference on Intelligent Engineering Systems, Miami, 2008, pp. 109 114.

\section{Authors' Profiles}

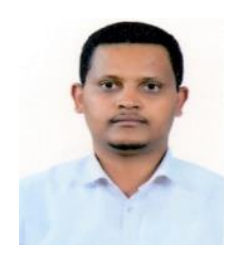

Wedajo T. Abdisa received his B.Sc. in Electrical Engineering (2008) from Addis Ababa University and his MEng degree (2018) from Engineering Institute of Technology, Australia. Since 2009 he has been working in the industrial control field as an electrical specialist, service engineer, engineering manager and as an industrial automation consultant. $\mathrm{He}$ is a certified programmer in Siemens S7 and PCS 7 Programmable Logic Controllers. His current research interests are application of Artificial Intelligence to solve problems or improve methods applied in the industrial automation field.

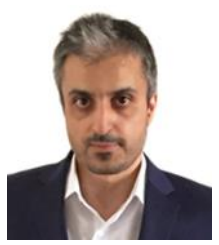

Hadi Harb holds a MEng (2000) in electrical-electronic engineering from the Lebanese University. He earned his MSc in 2001 and $\mathrm{PhD}$ in 2004 both in computer science from the Institut National des Sciences Appliquées INSA Lyon France, and the Ecole Centrale de Lyon France, respectively.

In 2004 he joined Centrale Lyon Innovation SA as a research engineer. In 2006 he founded and managed Ghanni, a company specialized in multimedia content recommendation and identification. Several European radio stations and websites licensed Ghanni's music recommendation technology. In 2015 he restructured Ghanni to transform it into a consultancy company in the domain of Artificial Intelligence and joined the Engineering Institute of Technology, Australia, as a lecturer. His current research interests are in the use of Artificial Intelligence techniques to solve industrial problems.
How to cite this paper: Wedajo T. Abdisa, Hadi Harb, "A Neural Network Based Motor Bearing Fault Diagnosis Algorithm and its Implementation on Programmable Logic Controller", International Journal of Intelligent Systems and Applications(IJISA), Vol.11, No.10, pp.1-14, 2019. DOI: 10.5815/ijisa.2019.10.01 